\section{THERAPEUTICS}

\section{Off-target efficacy}

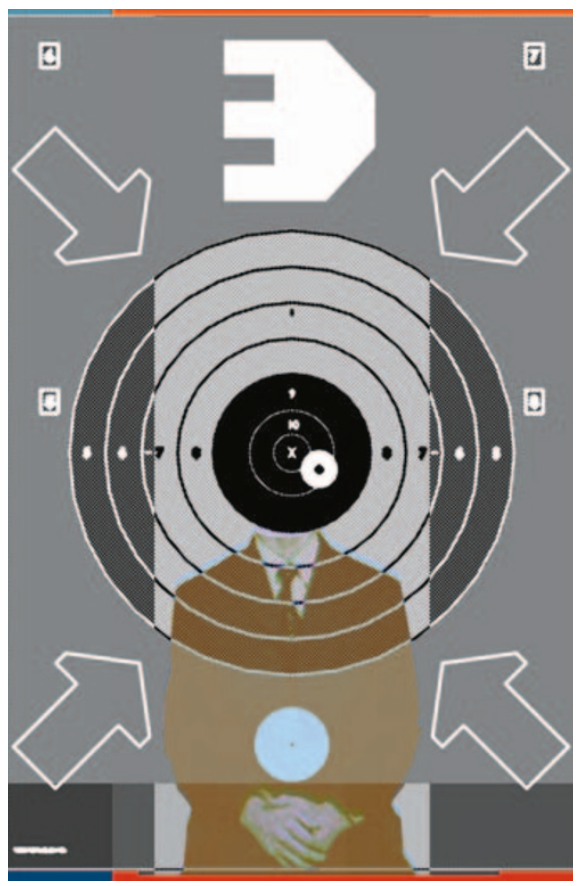

Farnesyltransferase (FTase) inhibitors (FTIs) were developed to inhibit oncogenic RAS, but FTIs have proved to be sporadically effective in vivo and independent of RAS activation. Lackner and colleagues have now discovered an off-target effect that might explain their mixed behaviour.

FTase is one of three prenyltransferases that are crucial for the post-translation modification of a wide range of proteins. Because FTase is closely related to geranylgeranyltransferase 1 (GGT1), inhibition of GGT1 by FTIs has been counter-screened. The structure of RAB geranylgeranyltransferase (RABGGT) was thought to be distinct from FTase and GGT1, making off targeting by FTIs unlikely.

The team from Bristol-Myers Squib and Exelixis had previously noted that several FTIs induced apoptosis through a mechanism that was independent of FTase or GGT1 inhibition. To understand the activity of these inhibitors better, they used the nematode worm Caenorhabditis elegans. Some germ cells in the worm spontaneously undergo apoptosis during maturation and the authors initially found that some of the FTIs are able to increase the numbers of apoptotic germ cells.

To identify potential new FTI targets, the team used a forward genetic chemical mutagenesis screen and a reverse genetic screen - RNA inhibition (RNAi) - to look for the gain or loss of genes that gave the same pro-apoptotic phenotype. Using the forward genetics approach the group identified 4 disrupted loci, and the one correlating with the highest levels of germ-cell apoptosis was analysed further. The mutated gene encoded a protein, VPS41, which is implicated in protein trafficking. From the RNAi knockdown screen the authors identified another five proteins that combine with VPS41 to produce the HOPS complex in yeast.

The HOPS complex, in combination with the RAB7 GTPase, drives protein trafficking through endosome-lysosome and autophagosome-lysomsome docking and fusion. Using RNAi to target $C$. elegans rab-7 and $r a b-5$, which are involved in protein trafficking and are modified by RABGGT, induced apoptosis in the germline cells. However, targeting of the homologues of the yeast autophagy-specific genes with RNAi did not, indicating that disruption of the endosome-lysosome function might be important for the pro-apoptotic response.

So, do the FTIs that induce apoptosis affect the RABGGT pathway? The authors show that inhibition of RABGGT activity in the worms increases the levels of apoptosis in response

\title{
Screen test for the stars
}

"Don't end up saying 'if only.' Get tested," says the US journalist Katie Couric of screening for colon cancer. However, as it is a complex and emotional issue, the promotion of cancer screening is controversial. The recent trend for celebrities to endorse personally relevant cancer-screening programmes has therefore come under scrutiny. Is it, in fact, a good thing, and does it actually have any impact?

To try and come up with some answers, Robin J. Larson and colleagues have analysed data taken from a randomdigit-dialling survey conducted in the United States during 2001-2002. Their results constitute the first data from a nationally representative sample of Americans of screening age who were asked about their exposure to, and the influence of, celebrity endorsements of cancer screening.
They report that, of those approached, $72 \%$ of people known to be eligible - that is, people of screening age and without a history of cancer - and $51 \%$ of people thought to be potentially eligible responded to the survey. This involved 360 women of 40 years or older and 140 men of 50 years or older. When asked if they 'had seen or heard a celebrity talk about' various screening programmes, most people responded positively: $73 \%$ of women for mammography, $63 \%$ of men for prostate-specific-antigen (PSA) testing, and $52 \%$ of adults for sigmoidoscopy or colonoscopy. In addition, of those people who had heard celebrity endorsements, the percentage who said they were more likely to undergo screening was $25 \%$ for mammography, $31 \%$ for PSA testing and $37 \%$ for sigmoidoscopy or colonoscopy.

The authors acknowledge that there are limitations to this study. For example, the study parameters mean that there are no data on whether celebrity endorsements encourage screening use in individuals for whom it would not be recommended. Furthermore, the authors are unable to ascertain whether the results were affected by the desire of respondents to give their perceived 'right answer' to the study questions.

On a personal level, undergoing cancer screening can have far-reaching consequences and should not be undertaken lightly. Partaking in informed and thoughtful discussions before making the decision to be screened is therefore increasingly being encouraged by organizations such as the US Preventative Services Task Force. The authors recommend that the provision of information, rather than persuasion, is the most appropriate approach to cancer screening, and they think that the persuasively emotive and one-sided pleas of celebrities to "Get tested" do not sit well with this view.

Lesley Cunliffe

(2) References and links

ORIGINAL RESEARCH PAPER Larson, R. J. et al. Celebrity endorsements of cancer screening. J. Natl Cancer Inst. 97. 693-695 (2005)

WEB SITE

The VA outcomes group: http://www.vaoutcomes.org/ 